\title{
New formulas for interferometric crosstalk penalty as a function of total crosstalk power, number of crosstalk contributions and signal extinction ratio
}

\author{
Rasmussen, Christian Jørgen; Jeppesen, Palle
}

Published in:

Proceedings on Lasers and Electro-Optics Society 2000 Annual Meeting

Link to article, DOI:

10.1109/LEOS.2000.890655

Publication date:

2000

Document Version

Publisher's PDF, also known as Version of record

Link back to DTU Orbit

Citation (APA):

Rasmussen, C. J., \& Jeppesen, P. (2000). New formulas for interferometric crosstalk penalty as a function of total crosstalk power, number of crosstalk contributions and signal extinction ratio. In Proceedings on Lasers and Electro-Optics Society 2000 Annual Meeting (Vol. 1, pp. 25-26). IEEE. https://doi.org/10.1109/LEOS.2000.890655

\section{General rights}

Copyright and moral rights for the publications made accessible in the public portal are retained by the authors and/or other copyright owners and it is a condition of accessing publications that users recognise and abide by the legal requirements associated with these rights.

- Users may download and print one copy of any publication from the public portal for the purpose of private study or research.

- You may not further distribute the material or use it for any profit-making activity or commercial gain

- You may freely distribute the URL identifying the publication in the public portal 


\title{
New Formulas for Interferometric Crosstalk Penalty as a Function of Total Crosstalk Power, Number of Crosstalk Contributions and Signal Extinction Ratio
}

\author{
Christian J. Rasmussen and Palle Jeppesen \\ Research Center COM, Technical University of Denmark, DK-2800 Lyngby, Denmark, \\ Phone: +45 45256352, Fax: +45 45936581, E-mail: cr@com.dtu.dk
}

\section{Introduction}

Interferometric crosstalk, also called incoherent crosstalk, occurs when reception of a desired signal is disturbed by undesired crosstalk contributions having the same wavelength as the desired signal but independent amplitudes and phases. This crosstalk type is known to be among the most destructive phenomena in optical networks owing to its accumulative nature and strong impact on the transmission quality. In many cases, interferometric crosstalk accumulation limits the network size [1,2], so it is of great importance to be able to assess the crosstalk impact accurately. Numerical models described in the literature [35] can be used for this but their complexity make them cumbersome in day-to-day work. Therefore it is felt that simple but still accurate penalty formulas are needed. In [6] we presented such formulas for the special case of manyin practice more than about 6 - crosstalk contributions. This earlier work has now been extended significantly by developing new formulas that apply to any number of crosstalk contributions. The new formulas state the crosstalk penalty as a function of the total crosstalk power, the number of contributions carrying this power and the signal extinction ratio. We consider both PIN and optically preamplified receivers. The authors know of no other published formulas which include the number of crosstalk contributions.

The crosstalk penalty formulas are empirical, and they are based on a numerical model. This model is described briefly along with its experimental verification before the formulas themselves are presented.

\section{Numerical model}

First step in the computation of the interferometric crosstalk penalty is the determination of the probability density function (pdf) of the total optical power $P$ at the receiver input at a given time instant. The overall bit error probability at optimum decision threshold is then found by combining this pdf and the conditional bit error probability for a given value of $P$. To find the latter probability, the decision variable in the receiver is assumed to be a Gaussian random variable whose mean is proportional to $P$, and whose variance is $k_{1}+k_{2} P$. Here $k_{1}$ is the thermal noise, and $k_{3} P$ is the signal-spontaneous emission beat noise (PIN receiver: $k_{2}=0$, optically preamplified receiver: $k_{2} P>>k_{l}$ ). The penalty is the crosstalk caused sensitivity reduction where the sensitivity is defined as the total average power into the receiver giving a bit error probability of $10^{-9}$.

A few comments should be made on the determination of the pdf of $P$. We consider the simultaneous reception of the desired signal with average power $P_{s}$ and $N$ independent crosstalk contributions each with an average power of $X P_{s} / N$ where $X$ is the total relative crosstalk level. $P$ can now be written as follows if it is assumed that signal and crosstalk have identical polarization states, identical extinction ratio $r$ and a mark density of 0.5 :

$$
P=P_{s}\left|s+\sum_{n-1}^{N} C_{n} e^{i \theta_{n}}\right|^{2}
$$

Here $s$ is $(2 /(1+r))^{0.5}$ or $(2 r /(1+r))^{0.5}$ depending on whether the desired signal is " 0 " or " 1 ". $C_{1} \ldots C_{N}$ are independent random variables which represent the amplitudes of the crosstalk contributions. With equal probability they take the values $(2 X /(N(1+r)))^{0.5}$ and $(2 X r /(N(1+r)))^{0.5}$. The phases of the crosstalk contributions are represented by the independent random variables $\theta_{1} \ldots \theta_{N}$ which are uniformly distributed over $[0 ; 2 \pi]$.

Figure 1 shows the computed penalty as a function of the total relative crosstalk level when the crosstalk power is evenly distributed among $1,2,4,6,10$ and an infinite number of crosstalk contributions. The upper graph is for a PIN receiver (extinction ratio $11.7 \mathrm{~dB}$ ), and the lower graph is for an optically preamplified receiver (extinction ratio $12.0 \mathrm{~dB}$ ). Naturally, the curves show that more crosstalk means higher penalty. But the penalty also increases when a given total crosstalk power is distributed among more crosstalk contributions. This increase saturates, however, at about 6 contributions. In addition to the dependencies illustrated in the figure, it was found that also the signal extinction ratio influences the crosstalk penalty significantly. This can be illustrated by the fact that the
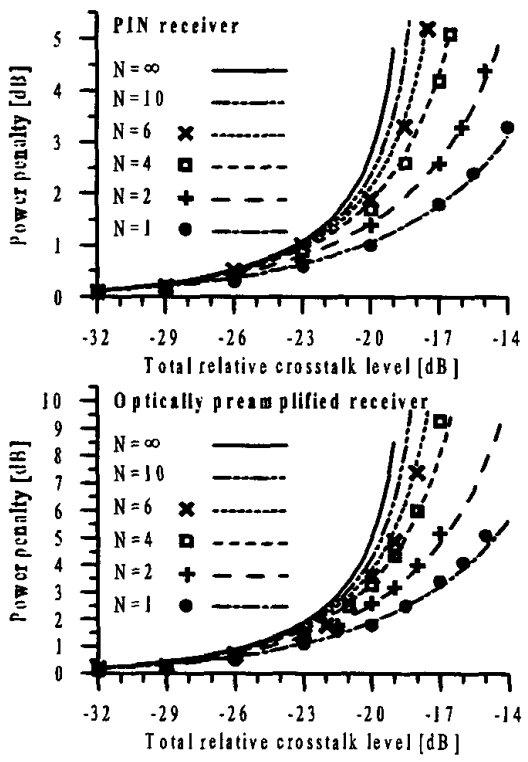

Figure 1: Computed(line) \& measured(symbol) penalty as a function of the total relative crosstalk level. The number of crosstalk contributions is parameter. 


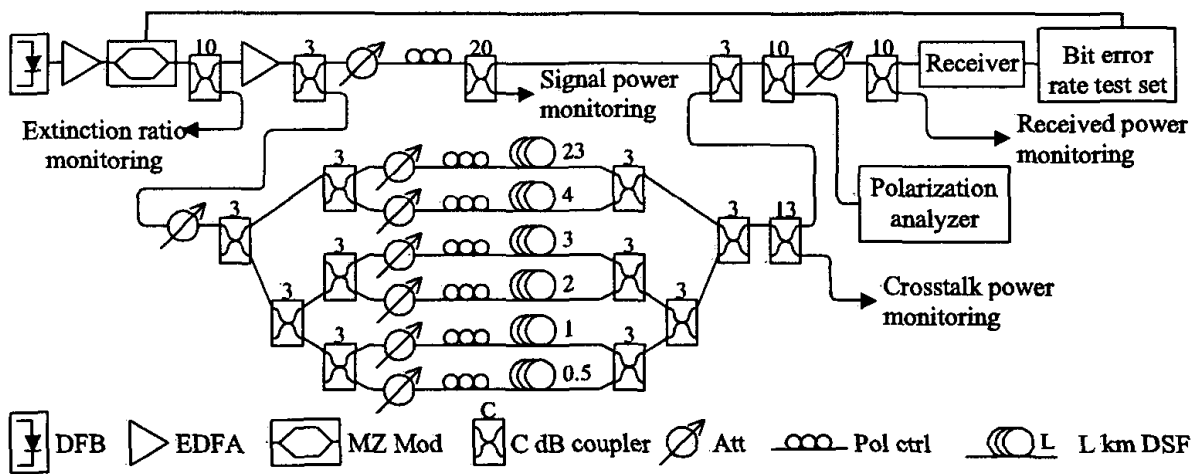

Figure 2: Experimental set-up for measurement of the penalty caused by interferometric crosstalk (MZ Mod: Mach-Zehnder modulator; Att: Attenuator; Pol ctrl: Polarization controller; DSF: Dispersion shifted fibre)

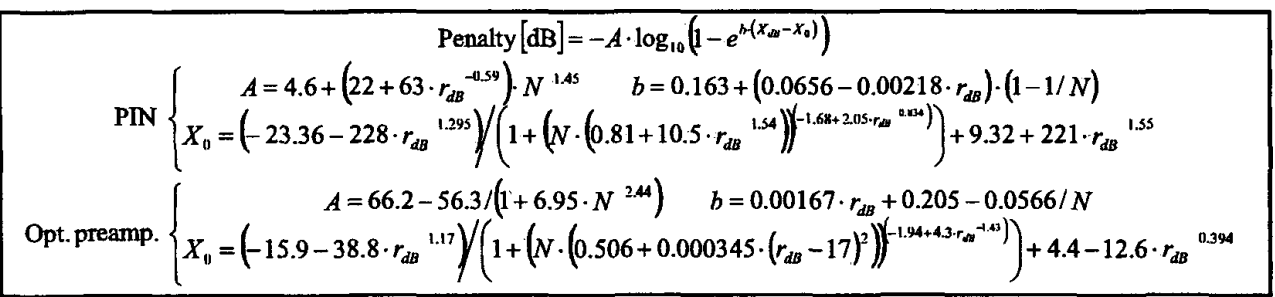

Figure 3: Formulas for the penalty caused by interferometric crosstalk.

total relative crosstalk level causing $1 \mathrm{~dB}$ penalty in a PIN receiver system infested by an infinite number of crosstalk contributions is $-27.1,-23.2$ and $-22.0 \mathrm{~dB}$ for extinction ratios of 6,12 and $20 \mathrm{~dB}$, respectively. Finally, it should be mentioned that the penalty for an optically preamplified receiver is roughly twice the penalty for a PIN receiver. The accuracy of this rule of thumb increases with decreasing extinction ratio. For extinction ratios below $12 \mathrm{~dB}$ and optically preamplified receiver penalties below $3 \mathrm{~dB}$, the accuracy is better than $0.3 \mathrm{~dB}$.

\section{Experimental verification}

To verify the numerical model, the crosstalk penalty was measured for $1,2,4$ and 6 crosstalk contributions in the setup shown in figure 2: CW light from a DFB laser with a linewidth of $48 \mathrm{MHz}$ was modulated with a $2^{31}-1$ PRBS at $5 \mathrm{Gbit} / \mathrm{s}$. The modulated signal was split into a desired signal part (follows the upper path) and a crosstalk part which is further split into six crosstalk contributions (follow the six lower paths). Six dispersion shifted fibres were used for decorrelation. Great care was taken to ensure that all crosstalk contributions had identical power at the input of the receiver, and that they had the same polarization state as the desired signal. Penalties were measured for both a PIN (11.7 dB extinction ratio) and an optically preamplified (12.0 dB extinction ratio) receiver. Measured and computed penalties are compared in figure 1 showing an excellent agreement that confirms the model.

\section{Empirical formulas}

Figure 3 displays empirical formulas that state the crosstalk penalty in PIN and optically preamplified receiver systems as a function of $r_{d B}=10 \log _{10}(r)$ (extinction ratio in $\mathrm{dB}$ ), $X_{d B}=10 \log _{10}(X)$ (total relative crosstalk level in $\mathrm{dB}$ ) and the number of crosstalk contributions which carry the crosstalk power, $N$. The formulas were developed as fits to penalties computed using the numerical model. The fitting is based on penalties computed for $r_{d \theta}=6,8, \ldots 20 \mathrm{~dB}, X_{d B}=33$, $-32.75, \ldots-12 \mathrm{~dB}$ and $N=1,2,3,4,6,10,20, \infty$. The investigated extinction ratio interval $[6 \mathrm{~dB} ; 20 \mathrm{~dB}]$ is expected to cover the range which is relevant for real world systems. Penalties given by the empirical formulas deviate less than $0.2 \mathrm{~dB}$ from the numerically computed penalties as long as the penalties are smaller than, respectively, $4 \mathrm{~dB}$ and $5 \mathrm{~dB}$ for PIN and optically preamplified receivers.

\section{Conclusion}

We present new formulas which are useful e.g. when designing optical networks since they make accurate computation of the transmission performance degradation due to interferometric crosstalk easy. The formulas state the crosstalk penalty as a function of the total crosstalk power the number of crosstalk contributions and the signal extinction ratio. Both PIN and optically preamplified receivers are considered. Penalties given by these formulas deviate less than $0.2 \mathrm{~dB}$ from penalties found using an experimentally verified numerical model.

\section{References}

[1] E. L. Goldstein, L. Eskildsen, A. F. Elrefaie. IEEEE Phot. Techn. Lett., 6(5):657-660, May 1994

[2] L. Gillner, C. P. Larsen, M. Gustavsson. Journal of Lightwave Technology, 17(1):58-67, January 1999

[3] S. L. Danielsen, C. Joergensen, B. Mikkelsen, K. E. Stubkjaer. IEEE Phot. Techn. Lett., 10(11):1635-1637, November 1998

[4] C. J. Rasmussen, F. Liu, R. J. S. Pedersen, B. F. Jørgensen, OFC'99 Technical Digest, TuR5

[5] T. Monroy, E. Tangdiongga, H. de Waardt. Journal of Lightwave Technology, 17(6):989-997, June 1999

[6] C. J. Rasmussen, F. Liu, P. Jeppesen: "Accurate Formulas for the Penalty caused by Interferometric Crosstalk". Accepted for ECOC2000. 